\title{
Zombie reviews taking over the PROSPERO systematic review registry. It's time to fight back!
}

Renato Andrade, Clínica do Dragão, Espregueira-Mendes Sports Centre - FIFA Medical Centre of Excellence, Porto, Portugal; Dom Henrique Research Centre, Porto, Portugal; Faculty of Sports, University of Porto, Porto, Portugal.

Rogério Pereira, Clínica do Dragão, Espregueira-Mendes Sports Centre - FIFA Medical Centre of Excellence, Porto, Portugal; Dom Henrique Research Centre, Porto, Portugal; Faculty of Sports, University of Porto, Porto, Portugal; University Fernando Pessoa, Porto, Portugal.

Adam Weir, MBBS, PhD, Sports Groin Pain Centre, Aspetar Orthopaedic and Sports Medicine Hospital, Doha, Qatar; Academic Medical Center, Amsterdam, The Netherlands.

Clare Ardern, Division of Physiotherapy, Linköping University, Linköping, Sweden; School of Allied Health, La Trobe University, Melbourne, Australia

João Espregueira-Mendes, Clínica do Dragão, Espregueira-Mendes Sports Centre - FIFA Medical Centre of Excellence, Porto, Portugal; Dom Henrique Research Centre, Porto, Portugal; Orthopaedics Department of Minho University, Braga, Portugal; 3B's Research Group-Biomaterials, Biodegradables and Biomimetics, University of Minho, Headquarters of the European Institute of Excellence on Tissue Engineering and Regenerative Medicine, AvePark, Parque de Ciência e Tecnologia, Zona Industrial da Gandra, 4805-017 Barco, Guimarães, Portugal; ICVS/3B's-PT Government Associate Laboratory, Braga/Guimarães, Portugal.

Corresponding author: Renato Andrade, Clínica do Dragão, Espregueira-Mendes Sports Centre FIFA Medical Centre of Excellence, Via Futebol Clube do Porto - F. C. Porto Stadium, Porto, Portugal; +351 220100 100; randrade@espregueira.com 
Prospective public registration of medical research is an important step to decrease the risk of duplication and reduce research waste, and to increase transparency ${ }^{1,2}$. It is a requirement for funding and publication of most clinical trials. Systematic reviews (SRs) are also important medical research - compiling original research to answer a specific research question using systematic searches, pre-established selection criteria, and critically appraising and synthesizing evidence to help answer a clinical question - helping clinicians make the best possible clinical decisions. Unfortunately, this cohesive advance in science is under attack. This editorial aims to highlight the current status of prospective registration of SRs and provide 5 useful tips.

\section{SYSTEMATIC REVIEWS REGISTRY}

The Centre for Reviews and Dissemination at the University of York hosts the international prospective registry platform for systematic reviews, known as PROSPERO (https://www.crd.york.ac.uk/prospero/) $)^{3}$. The registry is free to use for both those registering reviews and those consulting the register, web-based and straightforward; it takes less than one hour to complete a registry entry once the author has gathered all the necessary information ${ }^{4}$. Registering a SR involves navigating 22 mandatory and 18 optional fields that align with the Preferred Reporting Items for Systematic Reviews and Meta-Analyses (PRISMA) statement ${ }^{5}$. These fields cover the key methodological features of a SR that researchers need to consider for a good quality, clinically-useful SR .

A SR entry is only accepted for online registration after it has been evaluated by PROSPERO. The PROSPERO evaluation focuses on the completeness of the entry and its eligibility but does not include a consideration of the quality of the proposed review. After commencing a $\mathrm{SR}$, it is recognized that authors might need to amend their proposed methodology and protocol alterations may be required. For example, authors may start out thinking that a meta-analysis might be appropriate, but as the SR progresses, it becomes clear that the data are not suitable for meta-analysis). PROSPERO allows for (and encourages) authors to update their SR protocols as appropriate and to make these changes explicit. This promotes transparency and diminishes the risk of misleading results and spurious conclusions ${ }^{4,7}$. 


\section{PROSPERO - IS IT FILLING UP WITH ZOMBIE REVIEWS?}

Since PROSPERO was launched in February 2011, there have been more than 20,000 SR registrations. The number of new SR registrations has rapidly increased - almost doubling annually (Figure 1).

However, there is a problem with this apparent success in encouraging registrations. It might be tainted by a new phenomenon: zombie reviews. Part of the author's responsibility when registering a SR protocol is to keep the record updated as the SR progresses. Once the SR is complete, the PROSPERO registration should be updated to reflect completion and (ideally) publication of the SR. However, although the number of SRs registered in PROSPERO rose from 284 in 2011 to 10,819 in 2015, very few of these reviews appear to have been completed and published (Figure 2).

Only 7\% of SRs registered in PROSPERO between 2011 and 2015 have had their status updated to published. Putting aside the fact that some of these SRs might still be ongoing, there are two obvious explanations for this low conversion from SR protocol to published SR:

1. The SR has been abandoned, but the PROSPERO record has not been revised to show this

2. The SR has been completed and/or published but the PROSPERO record has not been updated

The problem is that abandoned SRs or SRs that have not been completed remain in PROSPERO indefinitely - as zombie titles - clogging up the registry (Figure 3). Updating the PROSPERO record allows new authors to avoid duplication of SRs that are still "alive", or to revive an abandoned SR.

We were curious about the presence of zombie reviews in sports medicine. We assessed 5 key musculoskeletal themes relevant to sports medicine on PROSPERO: anterior cruciate ligament, hamstring injuries, tendinopathy, patellofemoral pain and femoroacetabular impingement syndrome. Unfortunately, we found that zombie reviews do seem to have attacked sports medicine (Figure 4). 
PROSPERO aims to reduce waste in research by preventing duplication. Imagine you are planning a SR to determine the most effective loading protocols for the treatment of midportion Achilles tendinopathy. There are two important steps to take before you start:

1. Check the published literature to see if a high-quality SR has been recently published

2. Check PROSPERO to see if someone else has registered a SR that covers your question

In this example, after searching PROSPERO (search term "Achilles tendinopathy"), you would find 21 registered SRs with their status listed as ongoing (3 regarding loading protocols one registered in 2013 and two in 2017). So you might decide not to pursue your review to avoid duplication. However, if the PROSPERO records prove to be zombie reviews, it may mean that you do not pursue a quality review with excellent clinical utility.

\section{HOW CAN WE FIGHT BACK AGAINST ZOMBIE REVIEWS?}

PROSPERO has played vital role in the promotion of the best practice in conducting SRs, promoting quality and integrity in research ${ }^{2}$. Here are 5 recommendations that may help new and existing PROSPERO users to avoid adding to the problem:

- Before designing your SR methodology, search PROSPERO for protocols with similar research questions (but be cautious that some of the entries you find might be zombie reviews). In case of potential overlap, researchers should contact the author responsible for the protocol and to ask about the status of the SR

- Register your SR protocol on the PROSPERO database before conducting your database searches

- Once your SR reaches a point where it is discontinued (either completed or abandoned), update its registry entry, ideally within 4 weeks

- Whenever you make a modification to your methodology, update your PROSPERO record by outlining the modification and why you made it

- Update the PROSPERO record once the SR is published, ideally within 4 weeks

We suggest some changes that might be made at PROSPERO:

- Develop a system for systematically updating SR status, perhaps by sending annual emails, which must be responded to within a certain period of time (perhaps, 8 weeks). If no response is received, this could be flagged on the registry entry. 
- Seek funding to have a staff member(s) systematically search for PROSPERO unique identifiers that are reported or cited within published SRs, and update the PROSPERO entry publication status; this task could be included in quality journal feedback to PROSPERO when SRs are published

- Registrants should be informed of these processes when they register new SRs

\section{SUMMARY}

PROSPERO is an important resource for SR stakeholders, including researchers, peer reviewers, journal editors and guideline developers. Prospective registration and accurate, timely updating of the registry entry are vital to improve the quality of SRs in sports \& exercise medicine ${ }^{18}$. The system will grind to a halt if zombie reviews are not killed off. The BJSM publishes methodological tips and pearls for conducting systematic reviews. ${ }^{9}$

\section{REFERENCES}

1. Weir A, Rabia S, Ardern C Trusting systematic reviews and meta-analyses: all that glitters is not gold! Br J Sports Med 2016;50:1100-1101.

2. Davies S. The importance of PROSPERO to the National Institute for Health Research. Syst Rev 2012;1:5.

3. Booth $A$, Clarke $M$, Ghersi $D$, et al. An international registry of systematic-review protocols. Lancet 2011;377:108-109.

4. Chien PF, Khan KS, Siassakos D. Registration of systematic reviews: PROSPERO. BJOG 2012;119:903-905

5. Liberati A, Altman DG, Tetzlaff J, et al. The PRISMA statement for reporting systematic reviews and meta-analyses of studies that evaluate health care interventions: explanation and elaboration. PLoS Med 2009;6:e1000100

6 . loannidis J. The mass production of redundant, misleading, and conflicted systematic reviews and meta-analyses. Milbank Q 2016;94:485-514.

7. Higgins JP, Green S. Cochrane Handbook for Systematic Reviews of Interventions Version 5.1.0: The Cochrane Collaboration, 2011.

8. Moher D, Shamseer L, Clarke M, et al. Preferred reporting items for systematic review and meta-analysis protocols (PRISMA-P) 2015 statement. Syst Rev 2015;4:1

9. Ardern $\mathrm{CL}$ Systematic review hacks for the sports and exercise clinician: five essential methodological elements Br J Sports Med 2016;50:447-449. 


\section{ACKNOWLEDGMENTS}

The authors would like to sincerely thank and acknowledge Professor Mike Clarke for his valuable discussions and for critically reviewing the manuscript. Moreover, the authors would also like to thank Inês Barros (from @Caricatunhar) for her help in the creation and design of the figures.

\section{FIGURE LEGENDS}

Figure 1 - Incidence of new registrations and cumulative total of SR registrations in the PROSPERO database (as at 21st March 2017).

Figure 2 - Frequency and proportion of registered SR status in PROSPERO (assessed on 21 March 2017).

Figure 3 - Three possible pathways for a prospectively registered SR

Figure 4 - PROSPERO status of top- 5 themes in sports medicine (searches performed with filter [ti], as per 6 April 2017). 
18000

16000

14000

12000

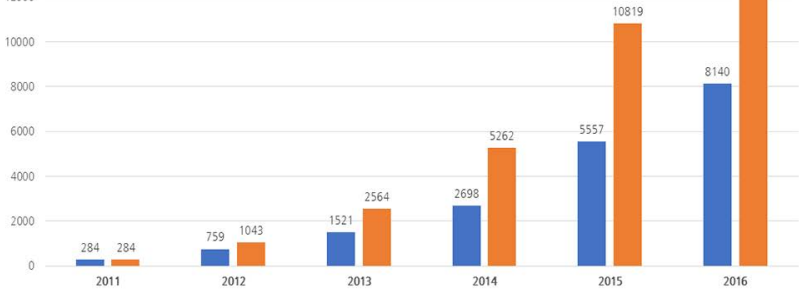

- New registries a Total registries 


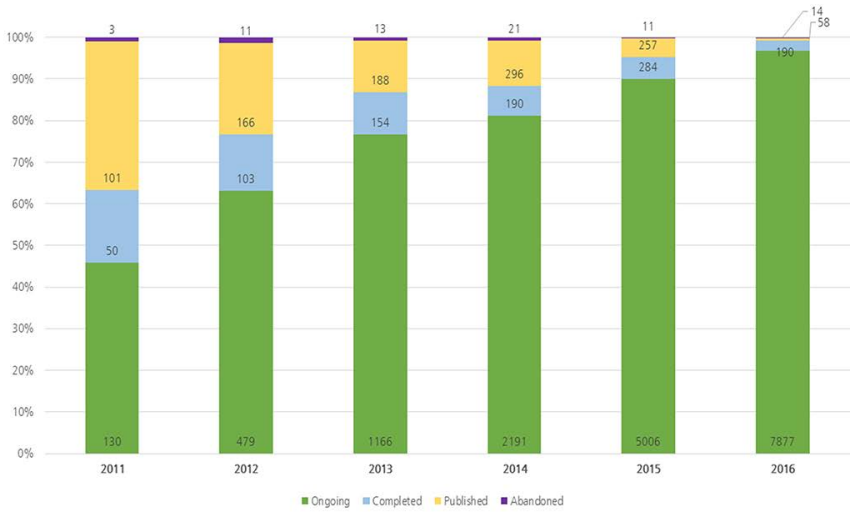




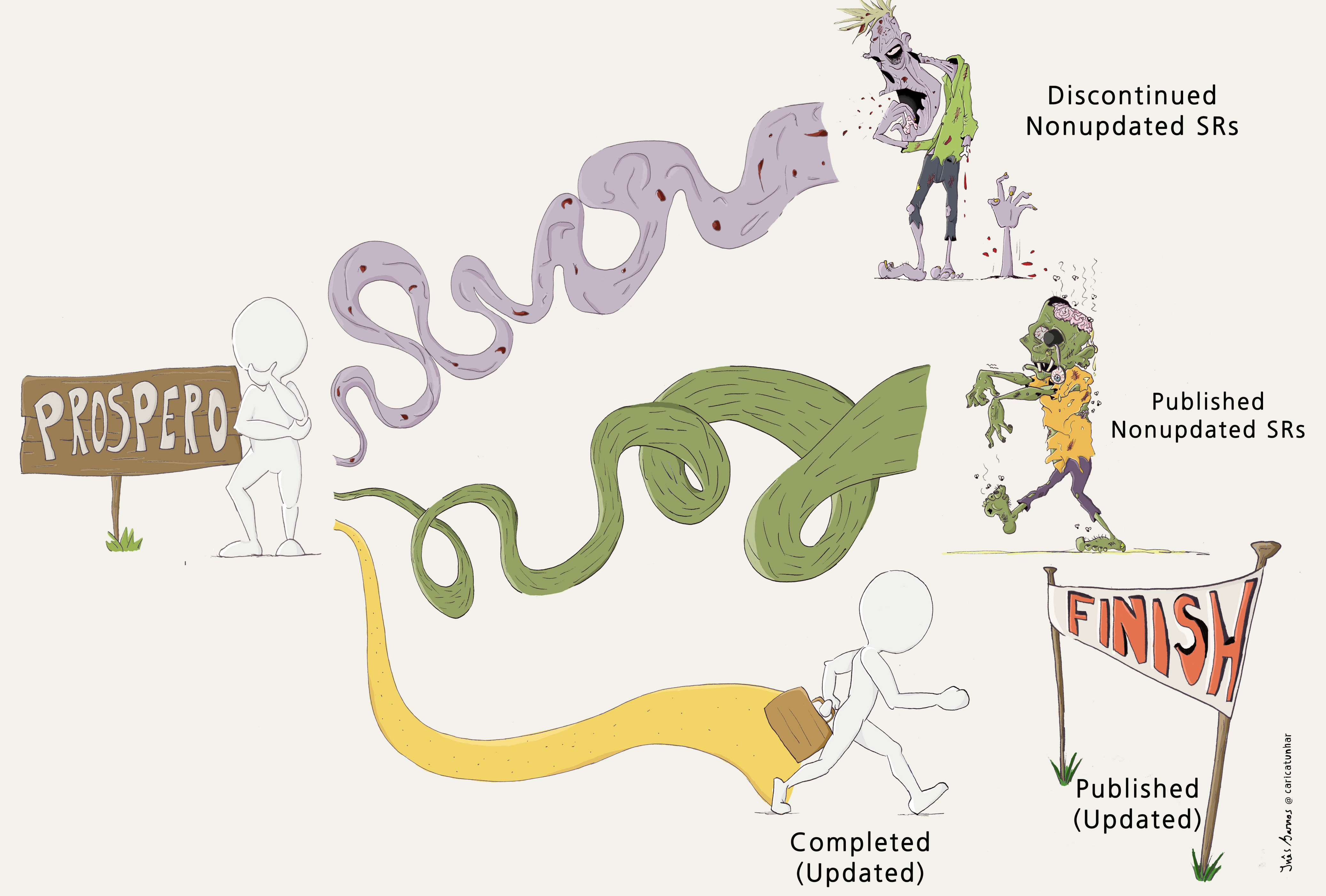




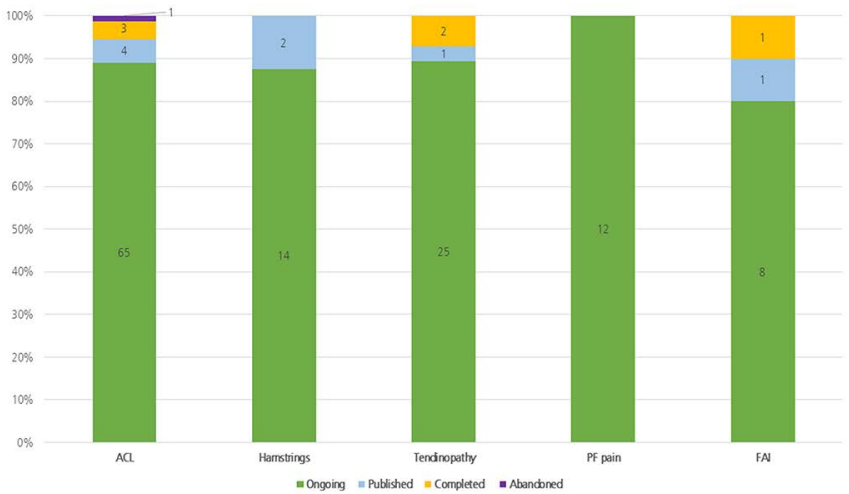

